\title{
Isquemia Miocárdica Silenciosa em Pacientes Submetidos à Prostatectomia Transuretral - Comparação entre Anestesia Subaracnóidea e Peridural *
}

\section{Silent Myocardial Ischaemia in Patients Undergoing Transurethral Resecti- on of Prostate - Comparison of Spinal Versus Epidural Anaesthesia}

Parshotam Lal Gautam ${ }^{1}$; Sunil Katyal ${ }^{1}$; Gurpreet Singh Wander ${ }^{2}$; Harpreet Kaur ${ }^{3}$

\section{RESUMO}

Gautam PL, Katyal S, Wander GS, Kaur H - Isquemia Miocárdica Silenciosa em Pacientes Submetidos à Prostatectomia Transuretral - Comparação entre Anestesia Subaracnóidea e Peridural

JUSTIFICATIVA E OBJETIVOS: A isquemia miocárdica silenciosa foi recentemente relacionada ao aumento de morbimortalidade cardíaca peri-operatória. Até $41 \%$ dos pacientes com doença coronariana conhecida ou fatores de risco cardíaco, submetidos à cirurgias não cardíacas, apresentaram isquemia peri-operatória. Vários autores compararam técnicas de anestesia regional e geral mas nenhum comparou o impacto de diferentes técnicas de anestesia no neuro-eixo na incidência e duração da isquemia miocárdica silenciosa. O objetivo deste estudo foi comparar duas técnicas diferentes de anestesia no neuro-eixo (subaracnóidea versus peridural) em pacientes idosos aleatoriamente selecionados e submetidos à prostatectomia transuretral. Optou-se por este grupo de pacientes idosos porque freqüentemente, apresentam doença coronariana silenciosa ou clinicamente aparente. Um outro fator importante que influenciou a escolha, foi a sobrecarga de volume e tremores causados pela prostatectomia transuretral nesses pacientes promovendo desequilíbrio entre consumo e oferta de oxigênio.

MÉTODO: Participaram deste estudo 40 pacientes submetidos a prostatectomia transuretral, que foram estudados em relação à isquemia miocárdica silenciosa com a ajuda de um equipamento Holter. A monitorização iniciou-se 1 hora antes da cirurgia, prosseguiu durante a cirurgia e após pelas próximas 24 horas. Os dados do Holter foram analisados por um DSM modelo 300.

RESULTADOS: A incidência geral de isquemia miocárdica silenciosa neste estudo foi de 30\%. Não foi estabelecida nenhuma relação entre isquemia miocárdica silenciosa e o tipo de anestesia. A maior parte dos episódios de isquemia miocárdica ocorreu no período pré-operatório e não tiveram

\footnotetext{
* Recebido do (Received from) do Departamento de Anestesiologia do Dayanand Medical College \& Hospital, Ludhiana, Punjab, India

1. Reader, Dept. of Anaesthesia

2. Professor, Dept. of Cardiology

3. PG Student, Dept. of Anaesthesia
}

Apresentado (Submitted) em 25 de agosto de 2003

Aceito (Accepted) para publicação em 04 de dezembro de 2004

Endereço para correspondência (Correspondence to)

Parshotam Lal Gautam, M.D.

61 Ashok Vihar, Rishi Nagar

Ludhiana, Punjab.

INDIA 141001

E-mail:plgautam@glide.net.in

(c) Sociedade Brasileira de Anestesiologia, 2004 relação com alterações hemodinâmicas. No entanto, a incidência e a gravidade de isquemia miocárdica silenciosa foi mais alta em pacientes com altos escores de Detsky, hipertensão arterial e anemia. Nenhum paciente apresentou efeitos cardíacos adversos.

CONCLUSÕES: O tipo de anestesia no neuro-eixo não influenciou a incidência de isquemia miocárdica silenciosa.

Unitermos: CIRURGIA: prostatectomia transuretral; DOENÇAS: isquemia miocárdica; TÉCNICAS ANESTÉSICAS: Regional, peridural, subaracnóidea

\section{SUMMARY}

Gautam PL, Katyal S, Wander GS, Kaur H - Silent Myocardial Ischaemia in Patients Undergoing Transurethral Resection of Prostate - Comparison of Spinal Versus Epidural Anaesthesia

BACKGROUND AND OBJECTIVES: In the recent past, silent myocardial ischaemia has been found to be associated with increased perioperative cardiac morbidity and mortality. As many as $41 \%$ of patients with either known coronary artery disease or with cardiac risk factors, undergoing a non-cardiac surgery, develop perioperative ischaemia. Various authors have compared regional techniques with general Anaesthesia in selected groups but no one has compared the impact of different techniques of centroneuraxial block on incidence and duration of silent myocardial ischaemia. Thus we compared two different techniques of centroneuraxial block (Spinal versus Epidural block) in aged patients selected randomly undergoing TURP surgery. We took TURP patients as they belong to geriatric age group and often have silent or apparent coexistent coronary artery disease. Secondly, TURP surgery related volume overload and shivering in these patients lead to further oxygen demand supply mismatch.

METHODS: Forty patients undergoing transurethral resection of prostate were monitored for perioperative silent myocardial ischaemia with the aid of a Holder device. Holter monitoring was started 1 hour prior to surgery and then continued during and after surgery the for next 24 hours. Holter data was analyzed using DSM model 300

RESULTS: The overall incidence of silent myocardial ischaemia in our study was $30 \%$. No relationship could be established between silent myocardial ischaemia and type of centroneuraxial block. Most of the episodes of myocardial ischaemia occurred in preoperative period and, these had no correlation with hemodynamic fluctuations. However the incidence and load of silent myocardial ischaemia was higher in-patients with high Detsky scoring, hypertension and anaemia. None of the patient had any adverse cardiac outcome.

CONCLUSIONS: Type of centroneuraxial block has no effect on incidence of silent myocardial ischaemia.

Key Words: ANESTHETIC TECHNIQUES, Regional: epidural, spinal block; DISEASES: myocardial ischaemia; SURGERY: tansurethral resection prostate 


\section{INTRODUÇÃO}

$\mathrm{E}^{\mathrm{r}}$ ventos cardíacos adversos são a principal causa de morbimortalidade após cirurgia não cardíaca em pacientes idosos. Muitos autores estudaram os fatores de risco peri-operatório e sistemas de classificação para avaliar populações em risco. A isquemia miocárdica silenciosa foi recentemente relacionada ao aumento de morbimortalidade cardíaca peri-operatória ${ }^{1}$. Até $41 \%$ dos pacientes com doença coronariana conhecida ou fatores de risco cardíaco, submetidos à cirurgias não cardíacas, apresentaram isquemia peri-operatória ${ }^{1-4}$. Além dos fatores de risco pré-operatórios, tipo de anestesia, alterações hemodinâmicas e estresse cirúrgico têm seu impacto no desequilíbrio entre consumo e oferta de oxigênio e morbidade cardíaca nesses pacientes.

Muitos autores compararam técnicas regionais e anestesia geral em grupos selecionados de pacientes com doença coronariana, em relação à incidência de isquemia miocárdica silenciosa ${ }^{5,6}$. Nenhum deles, porém, comparou o impacto do tipo de anestesia regional na incidência desta intercorrência. Existem resultados de alta incidência de isquemia miocárdica em pacientes submetidos à prostatectomia transuretral, provavelmente devido à idade avançada e doença coronariana preexistente. Além disso, a sobrecarga de volume e tremores relacionados à prostatectomia transuretral levam a um desequilíbrio entre o consumo e a oferta de oxigênio. $O$ objetivo deste estudo foi comparar duas técnicas diferentes de anestesia no neuro-eixo em pacientes idosos aleatoriamente selecionados submetidos à prostatectomia transuretral. O conhecimento da isquemia miocárdica peri-operatória em pacientes submetidos à cirurgias de rotina, como a prostatectomia transuretral, pode ajudar a calcular as implicações médicas e econômicas de estratégias preventivas. Utilizou-se o Holter começando no período pré-operatório e estendendo-se até 24 horas no pós-operatório.

\section{MÉTODO}

Após a aprovação da Comissão de Ética do hospital e assinatura de consentimento de participação, foram estudados de modo prospectivo 40 pacientes (idade $>50$ anos) submetidos à prostatectomia transuretral sob anestesia no neuro-eixo. Eles foram avaliados em relação à isquemia miocárdica com auxílio de ECG ambulatorial por 24 horas. Os pacientes foram aleatoriamente distribuídos em 2 grupos de 20, com base na técnica anestésica:

Grupo I recebeu anestesia subaracnóidea com $2 \mathrm{ml}$ de bupivacaína hiperbárica a $0,5 \%$;

Grupo II recebeu anestesia peridural com $15 \mathrm{ml}$ de lidocaína a $1,5 \%$.

Foi realizado um exame pré-anestésico completo para avaliar a presença de doença coronariana e outros fatores de risco associados. Todos os pacientes foram submetidos a exames de rotina, como hemograma, urina, glicemia de jejum, uréia, creatinina, eletrólitos, radiografia do tórax e ECG. O escore de Detsky foi aplicado para classificar risco cardíaco.
Os pacientes com bloqueio de condução intraventricular, padrão de insuficiência aos esforços do ventrículo esquerdo, segmento ST anormal em repouso no ECG e em uso de digoxina foram excluídos do estudo, além de pacientes com contra-indicações, que tenham recusado o método ou com anestesia inadequada ou ausente. Todos os pacientes receberam medicação pré-anestésica com 10 mg de diazepam oral na noite anterior e $5 \mathrm{mg}$ na manhã da cirurgia.

Os pacientes foram mantidos em jejum por 6 horas. Doses matinais de anti-hipertensivos e vasodilatadores coronarianos foram mantidas. Após a transferência para a mesa cirúrgica, foi inserido cateter venoso nas veias do dorso da mão para administração de solução fisiológica a 0,9\%. Freqüência cardíaca, pressão arterial não invasiva, ECG e saturação de oxigênio foram constantemente monitorizados durante o procedimento. Os pacientes do grupo I receberam anestesia subaracnóidea com agulha $23 \mathrm{G}$ na posição sentada. A punção foi realizada nos espaços $L_{2}-L_{3}$ ou $L_{3}-L_{4}$ e injetados $2 \mathrm{ml}$ de bupivacaína hiperbárica a $0,5 \%$. Foram a seguir colocados em decúbito dorsal e registrado o nível máximo de bloqueio sensitivo e sua duração. Os pacientes do grupo II receberam anestesia peridural com agulha Touhy $16 \mathrm{G}$ através da técnica de perda de resistência ao ar na seringa, nos espaços $L_{2}-L_{3}$ ou $L_{3}-L_{4}$. Foi inserido um cateter no espaço peridural. Uma injeção em bolus de $15 \mathrm{ml}$ de lidocaína a 1,5\% foi administrada pelo cateter peridural após uma dose teste de $3 \mathrm{ml}$. O cateter peridural foi fixado e os pacientes foram colocados em decúbito dorsal. Foram registrados o nível máximo e a duração do bloqueio sensitivo. Dose adicional de $3 \mathrm{ml}$ de lidocaína a $1,5 \%$ foi administrada no caso de anestesia insuficiente. Doses adicionais de $6 \mathrm{ml}$ foram administradas, quando necessárias.

As complicações intra-operatórias, como hipotensão arterial (PAS $<90$ ), foram tratadas com $3 \mathrm{mg}$ de mefenteramina venosa intermitente e bradicardia com 0,6 mg de atropina venosa. Ao final da cirurgia os pacientes foram enviados para a sala de recuperação pós-anestésica. Os eventos registrados no Holter foram analisados após 24 horas. Registrou-se eventos cardíacos peri-operatórios, como hipotensão arterial, disritmias, dor no peito etc. A recuperação do bloqueio sensitivo foi monitorizada e registrada. Os parâmetros do Holter foram analisados por um DSM modelo 300. Depressão do segmento ST acima de $1 \mathrm{~mm}$ foi considerada isquemia miocárdica silenciosa importante. Os dados foram estatisticamente analisados pelo teste $t$ de Student e o teste $Z$, isto é, teste de proporções e análise multivariável de regressão.

\section{RESULTADOS}

Os dados demográficos e o perfil clínico foram comparáveis entre os grupos, conforme demonstrado na tabela I. Não houve diferença significativa na incidência de fatores de risco cardiovascular nos dois grupos $(p<0,10)$. A duração do bloqueio sensitivo e os tempos de ressecção também foram semelhantes para os dois grupos. 
Tabela I - Comparação do Perfil Demográfico dos Dois Grupos Estudados

\begin{tabular}{|c|c|c|c|}
\hline \multirow[t]{2}{*}{ Características dos Pacientes } & \multicolumn{3}{|c|}{ Média } \\
\hline & Grupo I & Grupo II & Valores de $p$ \\
\hline Idade (anos) * & $69,25 \pm 7,70$ & $67,30 \pm 7,13$ & $>0,10$ \\
\hline Peso $(\mathrm{kg})$ * & $70,10 \pm 11,20$ & $73,70 \pm 9,65$ & $>0,10$ \\
\hline \multicolumn{4}{|l|}{ Estado físico } \\
\hline ASA I & 6 & 8 & $>0,10$ \\
\hline ASA II & 8 & 12 & $>0,10$ \\
\hline ASA III & 6 & & \\
\hline \multicolumn{4}{|l|}{ Diabete melito } \\
\hline Diabéticos & $17(85 \%)$ & $18(90 \%)$ & $>0,10$ \\
\hline Não diabéticos & $3(15 \%)$ & $2(10 \%)$ & \\
\hline \multicolumn{4}{|l|}{ Hipertensão arterial } \\
\hline Hipertensos & $10(50 \%)$ & $8(40 \%)$ & $>0,10$ \\
\hline Não hipertensos & $10(50 \%)$ & $12(60 \%)$ & \\
\hline \multicolumn{4}{|l|}{ Escore de Detsky } \\
\hline$<10$ & 14 & 18 & $>0,10$ \\
\hline 11 a 20 & 6 & 2 & $>0,10$ \\
\hline \multicolumn{4}{|l|}{ Exames pré-operatórios * } \\
\hline Hemoglobina & $11,51 \pm 1,77$ & $11,80 \pm 1,50$ & $>0,10$ \\
\hline \multicolumn{4}{|l|}{ Dados bioquímicos * } \\
\hline Uréia sangüínea & $42,20 \pm 44,38$ & $11,75 \pm 1,62$ & $>0,10$ \\
\hline Creatinina sérica & $1,56 \pm 0,87$ & $1,63 \pm 1,19$ & $>0,10$ \\
\hline Glicose sangüínea & $109,6 \pm 23,34$ & $113,10 \pm 22,58$ & $>0,10$ \\
\hline TG - Triglicérides & $156,35 \pm 95,51$ & $132,55 \pm 78,75$ & $<0,10$ \\
\hline HDL - Triglicérides & $39,25 \pm 8,08$ & $44,60 \pm 12,87$ & $>0,10$ \\
\hline LDL - Triglicérides & $107,00 \pm 23,69$ & $99,55 \pm 24,16$ & $>0,10$ \\
\hline VLDL - Triglicérides & $33,05 \pm 19,62$ & $33,555 \pm 17,52$ & $>0,10$ \\
\hline TL - Lipídeos totais & $636,35 \pm 146,23$ & $612,55 \pm 134,23$ & $>0,10$ \\
\hline
\end{tabular}

*Valores expressos em Média \pm D P

Na maioria dos casos, o tempo de ressecção foi de aproximadamente 20 minutos, exceto para quatro pacientes (dois em cada grupo), onde a ressecção ultrapassou uma hora. Desses quatro pacientes, um do Grupo I apresentou isquemia miocárdica silenciosa. Não houve diferença significativa na incidência de isquemia miocárdica nos dois grupos. Doze pacientes (30\%), com seis em cada grupo (Tabela II), apresentaram isquemia miocárdica silenciosa. O tempo de isquemia variou de 3 a 470 minutos no Grupo I, com duração média de 84 minutos, e no Grupo II variou de 20 a 479 minutos, com duração média de 201 minutos. O tempo de isquemia médio foi maior no grupo II.

A maioria dos episódios de isquemia miocárdica silenciosa ocorreu no período pré-operatório ( 5 de 6 pacientes no grupo I e 4 de 6 pacientes no grupo II). Foi observada isquemia miocárdica silenciosa pós-operatória em um paciente do grupo I e em dois pacientes do grupo II. Um paciente apresentou depressão do segmento ST 18 horas após a cirurgia, no grupo II. Esse paciente teve o escore de Detsky mais alto. Altos escores de Detsky também foram associados à episódios isquêmicos mais longos. A maioria dos pacientes com isque- mia miocárdica silenciosa apresentou hipertensão arterial (4 de 6 pacientes em cada grupo, ou $66,66 \%$ ). Diabete melito teve pouca relação com isquemia miocárdica silenciosa peri-operatória, neste grupo de pacientes. Alterações intra-operatórias na freqüência cardíaca e pressão arterial também não tiveram grande relação com episódios de depressão do segmento ST. Os pacientes com isquemia miocárdica silenciosa foram analisados através de análise multivariável para significância de vários fatores, isto é, idade, hipertensão arterial, diabete melito, perfil lipídico, escore de Detsky, anemia, tempo de ressecção, tratamento com beta-bloqueadores e tabagismo. Essa análise revelou que anemia seguida de hipertensão arterial foram os fatores mais importantes em pacientes com depressão do segmento ST (Tabela III, $p<0,05$ ).

Nos pacientes do grupo Il com isquemia miocárdica silenciosa, o escore de Detsky $(p<0,01)$ seguido da anemia $(p<0,05)$ e da hipertensão arterial $(p<0,05)$ foram estatisticamente significativos.

Os demais fatores não foram significativos para os dois grupos. 
Tabela II - Relação da Isquemia com as Características dos Pacientes, Período Peri-Operatório e Hemodinâmica Intra-Operatória

\begin{tabular}{|c|c|c|c|c|c|c|c|c|}
\hline & $\begin{array}{l}\text { Duração da } \\
\text { Isquemia (min) }\end{array}$ & FC & PA & $\begin{array}{l}\text { Depressão Máxima do } \\
\text { Segmento ST }(\mathrm{mm})\end{array}$ & $\begin{array}{c}\text { Período } \\
\text { Peri-Operatório }\end{array}$ & $\begin{array}{l}\text { Escore de } \\
\text { Detsky }\end{array}$ & $\mathrm{HT}$ & $\mathrm{DM}$ \\
\hline \multicolumn{9}{|l|}{ Grupo I } \\
\hline & 28 & 79 & $140 / 80$ & $-1,5$ & Pré-operatório & 10 & + & - \\
\hline & 3 & 65 & $130 / 80$ & $-2,0$ & Pré-operatório & 5 & + & - \\
\hline & 37 & 67 & $130 / 80$ & $-2,0$ & Pré-operatório & 0 & + & - \\
\hline & 8 & 80 & $150 / 90$ & $-2,0$ & Pré-operatório & 0 & + & - \\
\hline & 20 & 77 & $150 / 90$ & $-4,6$ & $\begin{array}{l}\text { Pós-operatório } \\
\text { (4 horas) }\end{array}$ & 15 & - & - \\
\hline & 410 & 95 & $140 / 90$ & $-2,9$ & Pré-operatório & 15 & - & - \\
\hline Média & 84 & & & & & & & \\
\hline \multicolumn{9}{|c|}{ Grupo II } \\
\hline & 20 & 80 & $140 / 80$ & $-1,6$ & Pré-operatório & 0 & + & - \\
\hline & 38 & 62 & $130 / 80$ & $-2,0$ & Pré-operatório & 0 & + & - \\
\hline & 470 & 90 & $140 / 80$ & $-2,8$ & Pré-operatório & 15 & - & - \\
\hline & 6 & 82 & $140 / 90$ & $-2,0$ & Pré-operatório & 0 & + & - \\
\hline & 198 & 85 & $160 / 100$ & $-2,1$ & $\begin{array}{l}\text { Pós-operatório } \\
\text { (18 horas) }\end{array}$ & 0 & - & - \\
\hline & 479 & 90 & $140 / 90$ & $-6,5$ & $\begin{array}{l}\text { Pós-operatório } \\
\text { (4 horas) }\end{array}$ & 20 & + & + \\
\hline Média & 201 & & & & & & & \\
\hline
\end{tabular}

Tabela III - Isquemia Silenciosa e Diferentes Variáveis

\begin{tabular}{|c|c|c|c|c|c|c|c|}
\hline \multirow[b]{2}{*}{ Variáveis } & \multicolumn{3}{|c|}{ Anestesia Subaracnóidea (Grupo I) } & \multicolumn{4}{|c|}{ Anestesia Peridural (Grupo II) } \\
\hline & Depressão ST & $\begin{array}{c}\text { Ordem de } \\
\text { Importância } \\
\mathrm{n}=20\end{array}$ & Normal & Depressão ST & $\begin{array}{l}\text { Ordem de } \\
\text { Importância } \\
n=20\end{array}$ & Normal & $\begin{array}{l}\text { Ordem de } \\
\text { Importância }\end{array}$ \\
\hline Idade & SI & & SI & SI & & SI & \\
\hline Diabete melito & $\mathrm{SI}$ & & SI & SI & & SI & \\
\hline Hipertensão arterial & $<0,05$ & 2 & SI & $<0,05$ & 3 & $\mathrm{SI}$ & 2 \\
\hline Perfil lipídico & SI & & SI & SI & & SI & \\
\hline Escore de Detsky & SI & & SI & $<0,01$ & 1 & $<0,05$ & 1 \\
\hline Anemia & $<0,05$ & 1 & $\mathrm{SI}$ & $<0,05$ & 2 & $<0,05$ & 3 \\
\hline Tempo de ressecção & $\mathrm{SI}$ & & $<0,05$ & SI & & $<0,05$ & \\
\hline Tratamento com $\beta$-Bloqueadores & SI & & SI & SI & & SI & \\
\hline Tabagismo & SI & & SI & $\mathrm{SI}$ & & SI & \\
\hline
\end{tabular}

SI (sem importância), $\mathrm{p}<0,001$ (altamente importante), $\mathrm{p}<0,05$ (importante)

\section{DISCUSSÃO}

O interesse na monitorização da isquemia miocárdica peri-operatória relaciona-se a possibilidade de ser usada como um previsor de eventos cardíacos adversos, tais como infarto do miocárdio, insuficiência cardíaca ou morte cardiovascular no período pós-operatório imediato, ou ainda como um indicador prognóstico de longo prazo. Vários fatores foram identificados para dimensionar o risco, em grupos sele- cionados, submetidos principalmente a grandes cirurgias vasculares. No entanto, como reações adversas graves são raras em cirurgias gerais de rotina, os resultados de grandes cirurgias vasculares não podem ser aplicados a pacientes submetidos a cirurgias não vasculares. A detecção de isquemia miocárdica silenciosa em grupos selecionados aleatoriamente pode ajudar a identificar pacientes com risco de desenvolverem isquemia miocárdica subclínica. Vários autores observaram uma relação entre isquemia miocárdica silenciosa e complicações cardiovasculares na população ci- 
rúrgica em geral ${ }^{1-3}$. Vários métodos foram usados para detectar isquemia miocárdica silenciosa precoce, entre eles ECG $^{7}$, Holter ${ }^{1,6,8-10}$, enzimas cardíacas como troponina le T, CPKMB ${ }^{11-13}$, cateter de artéria pulmonar ${ }^{14}$, ecocardiografia transesofágica ${ }^{15}$ e monitor de impedância elétrica miocárdica $^{16}$.

Este estudo verificou a incidência de isquemia miocárdica silenciosa numa população geriátrica selecionada aleatoriamente submetida à prostatectomia transuretral com dois tipos de anestesia regional usando ECG Holter ambulatorial e encontrou-se incidência geral de isquemia miocárdica silenciosa de $30 \%$. Nenhum paciente apresentou eventos adversos importantes. Vários autores relataram a incidência de eventos cardíacos adversos pós-operatórios após isquemia peri-operatória. Outros autores observaram uma incidência de $18 \%$ a $60 \%$ após técnicas de anestesia regional. Mangano e col. ${ }^{1}$ em 1990, e Windsor e col. ${ }^{6}$ em 1996 comparam duas técnicas anestésicas (subaracnóidea e geral) com relação à incidência de isquemia miocárdica silenciosa e não observaram diferença significativa entre elas. Neste estudo comparou-se duas técnicas de anestesia regional, subaracnóideae peridural. Aincidência de isquemia miocárdica silenciosa foi semelhante para os dois grupos. Não houve correlação importante entre alterações hemodinâmicas e isquemia miocárdica silenciosa. A duração e o nível do bloqueio também foram comparáveis entre os dois grupos. Aduração média do bloqueio foi $1 \frac{1}{2}$ horas e o nível médio foi $\mathrm{T}_{10}$ com o nível máximo em $\mathrm{T}_{8}$.

Amaioria dos autores observou isquemia miocárdica silenciosa pós-operatória coincidindo com a recuperação do bloqueio simpático. Variações na volemia, alterações da atividade adrenérgica, níveis de colinesterase plasmática, temperatura corporal, tremores, função pulmonar e percepção de dor foram considerados fatores contribuintes ${ }^{1,8}$. Ao contrário desses autores, observou-se neste estudo isquemia miocárdica silenciosa mais freqüente no período pré-operatório. Isso pode ter sido causado por ansiedade pré-operatória decorrente de medicação pré-anestésica inadequada do protocolo. Observou-se que a maioria dos pacientes estavam acordados durante o transporte para o centro cirúrgico.

Também estudou-se vários fatores de risco associados à isquemia miocárdica silenciosa, entre eles idade, diabete melito, hipertensão arterial, perfil lipídico, escore de Detsky, anemia, tempo de ressecção, terapia com beta-bloqueadores e tabagismo. Desses, a anemia e a hipertensão arterial foram os mais importantes no grupo I, e escore de Detsky seguido de anemia e hipertensão arterial foram os mais importantes no grupo II.

Como já foi estabelecido que isquemia miocárdica peri-operatória é produto do desequilíbrio entre consumo e oferta de oxigênio, talvez os pacientes com anemia e hipertensão arterial não tenham conseguido manter oferta adequada de oxigênio para o miocárdio em condições de estresse cirúrgico. Neste estudo, os pacientes dos dois grupos apresentaram importantes alterações de freqüência cardíaca e pressão arterial média no período pós-operatório imediato, com valo- res mais altos para os pacientes submetidos à anestesia peridural, no entanto, sem significância estatística. Para elucidar conclusivamente se as alterações hemodinâmicas não se correlacionam com isquemia miocárdica silenciosa e o papel dos vários fatores contribuintes são necessários estudos maiores.

Concluímos que, nas condições estudadas, os pacientes geriátricos com ou sem doença coronariana evidente, apresentam uma incidência geral de isquemia miocárdica silenciosa de aproximadamente $30 \%$. O tipo de anestesia regional (subaracnóidea ou peridural) não influenciou a incidência ou duração de isquemia miocárdica silenciosa. A maioria dos eventos isquêmicos foi observada no período pré-operatório. No entanto, são necessários outros estudos para determinar se esses curtos episódios de isquemia miocárdica silenciosa são importantes como prognóstico. A simples presença de episódios de isquemia miocárdica tem importância questionável na incidência de morbidade cardíaca grave, com já observado por Windsor e col. Outros fatores de risco para isquemia peri-operatória incluem escore de Detsky, hipertensão arterial e anemia.

\section{Silent Myocardial Ischaemia in Patients Undergoing Transurethral Resection of Prostate - Comparison of Spinal Versus Epidural Anaesthesia}

Parshotam Lal Gautam, M.D.; Sunil Katyal, M.D.; Gurpreet Singh Wander, M.D.; Harpreet Kaur, M.D.

\section{INTRODUCTION}

Adverse cardiac events are a major cause of morbidity and mortality after non-cardiac surgery in geriatric patients. Many authors have studied the perioperative risk factors and scoring systems to assess the populations at risk. In the recent past, silent myocardial ischaemia has been found to be associated with increased perioperative cardiac morbidity and mortality ${ }^{1}$. As many as $41 \%$ of patients with either known coronary artery disease or with cardiac risk factors, undergoing a non- cardiac surgery, develop perioperative ischaemia ${ }^{1-4}$. In addition to the preoperative cardiac risk factors, type of anaesthesia, hemodynamic fluctuations and surgical stress have their impact in causing myocardial oxygen demand supply mismatch and cardiac morbidity in these patients.

Most of the authors have compared regional techniques with general anaesthesia in selected group of patients suffering from coronary artery disease for silent myocardial ischaemia ${ }^{5,6}$. None of the author has compared the impact of type of regional block on the occurance of silent myocardial ischaemia. Patients undergoing transurethral resection of prostate (TURP) surgery have been reported to experience high inci- 
dence of myocardial ischaemia. This is probably due to geriatric age group and coexistent coronary artery disease in these patients. Secondly, TURP surgery related volume overload and shivering in these patients lead to further oxygen demand supply mismatch. Thus, we proposed to compare two different techniques of centroneuraxial block in randomly selected aged patients undergoing TURP surgery. Knowledge of perioperative myocardial ischaemia in these patients undergoing a routine surgery like TURP might help to estimate the medical and economic implication of preventive strategies. We used Holter monitoring starting from preoperative period and extending post operatively for 24 hours.

\section{METHODS}

Forty male patients (age > 50 years) undergoing elective TURP surgery under centroneuroaxial anaesthesia were prospectively studied for perioperative myocardial ischaemia using ambulatory ECG for 24 hours. After approval by hospital ethics committee, a written informed consent was obtained from all patients. Patients were randomly allocated into two groups of 20 each on the basis of anaesthetic technique:

Group I patients received centroneuraxial block by subarachnoid block with $2 \mathrm{~mL}$ heavy $0.5 \%$ bupivacaine;

Group II patients received centroneuraxial block by epidural block using $15 \mathrm{~mL}$ of lidocaine $1.5 \%$.

A through preanesthetic checkup was conducted to evaluate the patients for coronary artery disease and other associated risk factors. Routine investigations like haemogram, urine examination, fasting blood sugar, serum urea; serum creatinine; serum electrolytes, X-ray chest and ECG were done in all patients and recorded. Detsky scoring was done to stratify cardiac risk.

Patients with preoperative interventricular conduction block, left ventricular strain pattern, resting abnormal ST segment in ECG and on digoxin therapy were not included in the study. Patients who had either any contraindication to or refused the method, as well as those with inadequate or failed regional block were also excluded from the study. All patients were premedicated with tablet diazepam $10 \mathrm{mg}$ a night before and $5 \mathrm{mg}$ on the morning of surgery with a sip of water.

Patients were kept fasting for 6 hours prior to surgery. Morning doses of antihypertensive and antianginal drugs were continued with a sip of water, if any three leads Holter monitoring was started preoperatively on the morning of surgery and was continued perioperatively for a total period of 24 hours. After shifting the patients on operation table, intravenous catheter was inserted in the veins on the dorsum of hand and connected to free running saline infusion. Heart rate, non-invasive blood pressure, ECG and oxygen saturation were monitored continuously intraoperatively.

Group I patients received subarachnoid block with 23G L.P. needle in sitting position at $\mathrm{L}_{2}-\mathrm{L}_{3}$ or $\mathrm{L}_{3}-\mathrm{L}_{4}$ intervertebral spaces with $2 \mathrm{~mL}$ heavy $0.5 \%$ bupivacaine injection. Patients were made to lie supine and levels of maximum sensory block and duration were recorded. Group II patients received epidural block with 16G Touhy's needle using loss of resistance technique at $L_{2}-L_{3}$ or $L_{3}-L_{4}$ intervertebral spaces. Epidural catheter was placed in epidural space. Lidocaine $1.5 \%, 15 \mathrm{~mL}$ was injected as a bolus after a test dose of $3 \mathrm{~mL}$ through epidural catheter. Epidural catheter was secured and patients were placed in supine positions. Maximum level and duration of sensory block were recorded. Incremental dose of lidocaine $1.5 \% 3 \mathrm{~mL}$ was administered in case of inadequate effect and top up of $6 \mathrm{~mL}$ was repeated as and when required.

Intraoperative complications like hypotension (systolic blood pressure $<90$ ) were treated with intermittent mephenteramine $3 \mathrm{mg} \mathrm{I}$.V. injection and bradycardia with atropine $0.6 \mathrm{mg}$ I.V injection. After completion of surgery, patients were referred to recovery room. After 24 hours, record of events on Holter monitoring was obtained and analyzed. Perioperative cardiac events, like episodes of hypotension, arrhythmia, chest paint etc. were recorded. Weaning of sensory block was monitored and recorded. Parameters recorded on Holter monitoring were observed and analyzed using DSM model 300. ST segment depression greater than $1 \mathrm{~mm}$ were considered as significant silent myocardial ischaemia. Statistical analysis was performed using Student's $t$ test, Z-test i.e. test of proportions and multivariate logistic regression analysis.

\section{RESULTS}

Demographic data and clinical profile were comparable among two groups as shown (Table I). There was no significant difference in the incidence of cardiovascular risk factors in both the groups ( $p$ value, $<0.10$ ). Duration of sensory block and resection times were also similar in both the groups. In most of the cases, resection time was approximately 20 minutes, except for four patients (two in each group), where resection time crossed one hour. Out of these four patients, one patient of Group I had silent myocardial ischaemia. There was no significant difference in incidence of myocardial ischaemic episodes in both the groups. Twelve patients $(30 \%)$ with six in each group (Table II), were found to have silent myocardial ischaemia. The ischaemic load in group I varied from 3 minutes to 470 minutes, with mean duration of 84 minutes, and in group II varied from 20 minutes to 479 minutes, with mean duration of 201 minutes. Mean ischaemic load was observed to be higher in group II.

It was also observed that most of myocardial silent ischaemic episodes occurred in preoperative period (i.e. 5 out of 6 in group I and 4 out of 6 in-group II). Postoperative silent ischaemia occurred in one patient in spinal group and two patients in epidural group. One patient had ST segment depression on Holter monitoring as late as 18 hours after surgery in study group II. That patient had the highest Detsky score. Also, high Detsky scores were found to be associated with longer silent ischaemic episodes. Most of the patients with silent myocardial ischaemic changes were 
Table I - Comparison of Demographic Profile of Two Study Groups

\begin{tabular}{|c|c|c|c|}
\hline \multirow[t]{2}{*}{ Patient Characteristics } & \multicolumn{3}{|c|}{ Mean Values } \\
\hline & Group I & Group II & $p$ values \\
\hline Age (year) * & $69.25 \pm 7.70$ & $67.30 \pm 7.13$ & $>0.10$ \\
\hline Weight $(\mathrm{kg})^{*}$ & $70.10 \pm 11.20$ & $73.70 \pm 9.65$ & $>0.10$ \\
\hline \multicolumn{4}{|l|}{ Physical Status } \\
\hline ASAI & 6 & 8 & $>0.10$ \\
\hline ASA II & 8 & 12 & $>0.10$ \\
\hline ASA III & 6 & & \\
\hline \multicolumn{4}{|l|}{ Diabetes mellitus } \\
\hline Diabetic & $17(85 \%)$ & $18(90 \%)$ & $>0.10$ \\
\hline Non diabetic & $3(15 \%)$ & $2(10 \%)$ & \\
\hline \multicolumn{4}{|l|}{ Arterial hypertension } \\
\hline Hypertensive & $10(50 \%)$ & $8(40 \%)$ & $>0.10$ \\
\hline Non hypertensive & $10(50 \%)$ & $12(60 \%)$ & \\
\hline \multicolumn{4}{|l|}{ Detsky score } \\
\hline$<10$ & 14 & 18 & $>0.10$ \\
\hline 11 to 20 & 6 & 2 & $>0.10$ \\
\hline \multicolumn{4}{|c|}{ Preoperative Investigations * } \\
\hline Hemoglobin & $11.51 \pm 1.77$ & $11.80 \pm 1.50$ & $>0.10$ \\
\hline \multicolumn{4}{|l|}{ Biochemical Data * } \\
\hline Blood urea & $42.20 \pm 44.38$ & $11.75 \pm 1.62$ & $>0.10$ \\
\hline Serum creatinine & $1.56 \pm 0.87$ & $1.63 \pm 1.19$ & $>0.10$ \\
\hline Blood sugar & $109.6 \pm 23.34$ & $113.10 \pm 22.58$ & $>0.10$ \\
\hline S.TGs & $156.35 \pm 95.51$ & $132.55 \pm 78.75$ & $<0.10$ \\
\hline S.HDL & $39.25 \pm 8.08$ & $44.60 \pm 12.87$ & $>0.10$ \\
\hline S.LDL & $107.00 \pm 23.69$ & $99.55 \pm 24.16$ & $>0.10$ \\
\hline S.VLDL & $33.05 \pm 19.62$ & $33.555 \pm 17.52$ & $>0.10$ \\
\hline S.TLs & $636.35 \pm 146.23$ & $612.55 \pm 134.23$ & $>0.10$ \\
\hline
\end{tabular}

* Values expressed in Mean \pm SD

found to have concomitant hypertension (i.e. 4 out of 6 in each group or $66.66 \%$ ) Diabetes mellitus was seen to be poorly related with perioperative silent myocardial ischaemia. Intraoperative changes in the heart rate and blood pressure were also not significantly linked with episodes of ST depression. Patients with silent ischaemia were analysed using multivariate analysis for significance of various factors i.e. age, arterial hypertension, diabetes mellitus, lipid profile, Detsky score, anaemia, resection time, beta-blocker treatment and smoking. From this analysis, it was found that in patients showing ST depression in group I, anaemia followed by hypertension were the most significant factors (Table III, $p<0.05$ ).

In patients showing silent ischaemia in group II, Detsky scoring $(p<0.01)$ followed by anaemia $(p<0.05)$ and arterial hypertension $(p<0.05)$ were found to be statistically significant.

Rest of the factors was observed to be non-significant in both groups.

Revista Brasileira de Anestesiologia

Vol. 54, N 4, Julho - Agosto, 2004

\section{DISCUSSION}

The interest in the monitoring for perioperative myocardial ischaemia reflects the possibility of using this as a predictor of adverse cardiac outcomes such as myocardial infarction, heart failure or cardiovascular death in early postoperative period or as a long-term prognostic indicator. Anumber of risk factors have been identified to stratify risk in selected population groups undergoing mainly major vascular surgeries. However, as hard adverse reaction are rare in routine general surgeries, those results of major vascular surgeries can not be applied to patients undergoing non-vascular surgeries. Detection of silent myocardial ischaemia in these population groups selected at random may help to identify the patient at risk of developing subclinical myocardial ischaemia. In general surgical population, number of authors have found relationship between silent myocardial ischaemia and adverse cardiovascular complications and their outcome ${ }^{1-3}$. Various 
Table II - Relationship of Ischaemic Load with Patient Characteristics, Perioperative Period and Intraoperative Hemodynamics

\begin{tabular}{|c|c|c|c|c|c|c|c|c|}
\hline & $\begin{array}{l}\text { Duration of } \\
\text { Ischaemia } \\
\text { (min) }\end{array}$ & $\mathrm{HR}$ & $\mathrm{BP}$ & $\begin{array}{l}\text { Maximum Depression of } \\
\text { ST Segment }(\mathrm{mm})\end{array}$ & $\begin{array}{l}\text { Perioperative } \\
\text { Period }\end{array}$ & Detsky Score & HT & $\mathrm{DM}$ \\
\hline \multicolumn{9}{|c|}{ Group I } \\
\hline & 28 & 79 & $140 / 80$ & -1.5 & Preoperative & 10 & + & - \\
\hline & 3 & 65 & $130 / 80$ & -2.0 & Preoperative & 5 & + & - \\
\hline & 37 & 67 & $130 / 80$ & -2.0 & Preoperative & 0 & + & - \\
\hline & 8 & 80 & $150 / 90$ & -2.0 & Preoperative & 0 & + & - \\
\hline & 20 & 77 & $150 / 90$ & -4.6 & $\begin{array}{l}\text { Postoperative } \\
\text { (4 hours ) }\end{array}$ & 15 & - & - \\
\hline & 410 & 95 & $140 / 90$ & -2.9 & Preoperative & 15 & - & - \\
\hline Mean & 84 & & & & & & & \\
\hline \multicolumn{9}{|c|}{ Group II } \\
\hline & 20 & 80 & $140 / 80$ & -1.6 & Preoperative & 0 & + & - \\
\hline & 38 & 62 & $130 / 80$ & -2.0 & Preoperative & 0 & + & - \\
\hline & 470 & 90 & $140 / 80$ & -2.8 & Preoperative & 15 & - & - \\
\hline & 6 & 82 & $140 / 90$ & -2.0 & Preoperative & 0 & + & - \\
\hline & 198 & 85 & $160 / 100$ & -2.1 & $\begin{array}{l}\text { Postoperative } \\
\text { (18 hours) }\end{array}$ & 0 & - & - \\
\hline & 479 & 90 & $140 / 90$ & -6.5 & $\begin{array}{l}\text { Postoperative } \\
\text { (4 hours) }\end{array}$ & 20 & + & + \\
\hline Mean & 201 & & & & & & & \\
\hline
\end{tabular}

Table III - Silent Ischaemia and Different Variables

\begin{tabular}{|c|c|c|c|c|c|c|c|}
\hline \multirow[b]{2}{*}{ Variables } & \multicolumn{3}{|c|}{ Spinal Anesthesia (Group I) } & \multicolumn{3}{|c|}{ Epidural Anesthesia (Group II) } & \multirow[b]{2}{*}{$\begin{array}{c}\text { Order of } \\
\text { Significance }\end{array}$} \\
\hline & ST Depression & $\begin{array}{c}\text { Order of } \\
\text { Significance } \\
n=20\end{array}$ & Normal & ST Depression & $\begin{array}{c}\text { Order of } \\
\text { Significance } \\
n=20\end{array}$ & Normal & \\
\hline Age & NS & & NS & NS & & NS & \\
\hline Diabetes mellitus & NS & & NS & NS & & NS & \\
\hline Arterial hypertension & $<0.05$ & 2 & NS & $<0.05$ & 3 & NS & 2 \\
\hline Lipid profile & NS & & NS & NS & & NS & \\
\hline Detsky score & NS & & NS & $<0.01$ & 1 & $<0.05$ & 1 \\
\hline anaemia & $<0.05$ & 1 & NS & $<0.05$ & 2 & $<0.05$ & 3 \\
\hline Resection time & NS & & $<0.05$ & NS & & $<0.05$ & \\
\hline$\beta$-Blocker treatment & NS & & NS & NS & & NS & \\
\hline Smoking & NS & & NS & NS & & NS & \\
\hline
\end{tabular}

NS - Non-significant, $p<0.01$ (highly significant), $p<0.05$ (significant)

modalities have been used in different studies to detect early silent myocardial ischaemia.

These include ECG ${ }^{7}$, Holter monitoring ${ }^{1,6,8-10}$, cardiac enzyme levels like Troponin I and T, CPKMB ${ }^{11-13}$, pulmonary artery catheter ${ }^{14}$, transesophageal echocardiography ${ }^{15}$ and myocardial electrical impedance monitor ${ }^{16}$.

In our study, we studied perioperative silent myocardial ischaemia in geriatric population selected randomly undergoing TURP surgery under two types of centroneuraxial block using Holter ambulatory ECG we found an overall silent ischaemia incidence of $30 \%$. None of the patient had any major untoward event. The incidence of postoperative adverse cardiac events following perioperative ischaemia has been found to be related to silent ischaemia in various studies. Different authors have reported incidence varying between $18 \%-60 \%$ after regional anaesthetic techniques in previous studies. Mangano et al ${ }^{1}$ in 1990 and Windsor et al ${ }^{6}$ in 1996 compared two anaesthetic techniques (spinal and general anaesthesia) for incidence of silent myocardial ischaemia and demonstrated no significant difference between them. In Vol. 54, No 4, Julho - Agosto, 2004 
our study, we compared two regional anaesthetic techniques: spinal and epidural Anaesthesia. The incidence of silent myocardial ischaemia was similar in both groups. No significant correlation could be established between the hemodynamic fluctuations and silent myocardial ischaemia. Duration and level of blockade were also comparable between the two study groups. The average duration of block was $1 \frac{1}{2}$ hours and average level up to T10 with maximum level up to T8.

In most of the studies researchers have found silent myocardial ischaemia in the postoperative period which coincided with the cessation of sympathetic block. The shifting of fluids in the central compartment, changes in adrenergic activity, plasma cholinesterase levels, body temperature, shivering, pulmonary function and perception of pain are found to be the contributing factors ${ }^{1,8}$. Contrary to these authors, we found that silent myocardial ischaemia occurred more often in preoperative period in our patients. This could be due to preoperative anxiety because of inadequate premedication as per our protocol. We found that most of our patients were wide-awake while being wheeled in the operation theatre.

In our study, we also studied various risk factors associated with silent myocardial ischaemia. These included age, diabetes mellitus, arterial hypertension, lipid profile, Detsky score, anaemia, resection time, beta-blocker therapy and smoking. Out of these, anaemia and arterial hypertension were found to be most significant in group I patients while Detsky score followed by anaemia and arterial hypertension were found to be most significant in group II patients.

As it is established that perioperative myocardial ischaemia is a product of oxygen demand supply mismatch, probably patient with anaemia and hypertension failed to maintain adequate myocardial oxygen supply in stressful perioperative conditions. In our study, patients in both study groups showed significant variations in heart rate as well as in mean arterial pressure in early postoperative period, with higher values of these in patients under epidural anaesthesia. However, no statistical significance was found. However, to elucidate conclusively whether haemodynamic drifts have poor correlation with myocardial ischaemia and role of various contributing factors, a substantially large planned study is required.

In summary, we conclude that the geriatric patients with or without evident coronary artery disease had overall incidence of silent myocardial ischaemia in range of $30 \%$. The type of regional Anaesthesia (spinal or epidural) did not influence the incidence or load of silent myocardial ischaemia. Most of the ischaemic events were found to occur preoperatively. Secondly, whether these short episodes of silent ischaemia have been significant in the prognostic prediction needs further longitudinal supervised trials. Merely the presence of myocardial ischaemic episodes have questionable role to cause significant cardiac morbidity as reported earlier by Windsor et al. Various risk factors for perioperative ischaemia include high Detsky score, hypertension and anaemia.

\section{REFERÊNCIAS - REFERENCES}

01. Mangano DT, Browner WS, Hollenberg M et al - Perioperative ischaemia research group. association of perioperative myocardial ischaemia with cardiac morbidity and mortality in men undergoing non cardiac surgery. N Eng J Med, 1990;323: 1781-1788.

02. McCann RL, Clements FM - Silent myocardial ischaemia in patients undergoing peripheral vascular surgery: incidence and association with perioperative cardiac morbidity and mortality. J Vasc Surg, 1989;9:583-587.

03. Mangano DT, Hollenberg M, Fegert G et al - Perioperative myocardial ischaemia in patients undergoing noncardiac surgery: I. Incidence and severity during the 4 day perioperative period. The study of perioperative ischaemia (SPI) research group. J AM Coll Cardiol, 1991;17:843-850.

04. Mangano DT, Wong MG, London MJ et al - Perioperative myocardial ischaemia in patients undergoing noncardiac surgery: II. Incidence and severity during the $1^{\text {st }}$ week after surgery. The study of perioperative ischaemia (SPI) research group J AM Coll Cardiol, 1991;17:851-857.

05. Lawson RA, Turner WH, Reeder MK at al - Haemodynamic effects of transurethral prostatectomy. Br J Urol, 1993;72:74-79.

06. Windsor A, French GW, Sear JW et al - Silent myocardial ischaemia in patients undergoing transurethral prostatectomy. A study to evaluate risk scoring and anaesthetic technique with outcome. Anaesthesia, 1996;51:728-732.

07. Blackburn H, Keys A, Simonson E et al - The electrocardiogram in population studies. A classification study. Circulation, 1960;21:1160-1175.

08. Landesberg G, Luria MH, Cotev S et al - Importance of long-duration postoperative ST- segment depression in cardiac morbidity after vascular surgery. The Lancet, 1993;341:715-719.

09. Edwards ND, Alford AM, Dobson PM et al - Myocardial ischaemia during tracheal intubation and extubation. $\mathrm{Br} \mathrm{J}$ Anaesth, 1994;73:537-539.

10. Leung JM, Voskanian A, Bellows WH et al - Automated electrocardiograph ST segment trending monitors: accuracy in detecting myocardial ischaemia. Anesth Analg, 1998;87:4-10.

11. Metzler H, Gries M, Rehak P et al - Perioperative myocardial cell injury: the role of troponins. Br J Anaesth, 1997;78:386-390.

12. Noble JS, Reid AM, Jordan LV et al - Troponin I and myocardial injury in the ICU. Br J Anaesth, 1999;82:41-46.

13. Neill F, Sear JW, French G et al - Increases in serum concentrations of cardiac proteins and the prediction of early postoperative cardiovascular complications in noncardiac surgery patients. Anaesthesia, 2000;55:641-647.

14. Kaplan JA, Wells PH - Early diagnosis of myocardial ischaemia using the pulmonary arterial catheter. Anesth Analg, 1981;60:789-793.

15. Ellis JE, Shah MN, Briller JE et al - A comparison of methods for the detection of myocardial ischaemia during noncardiac surgery: automated ST-segment analysis systems, electrocardiography and transesophageal echocardiography. Anesth Analg, 1992;75:764-772.

16. Howie MB, Dzwonczyk R, McSweeney TD - An evaluation of a new two-electrode myocardial electrical impedance monitor for detecting myocardial ischaemia. Anesth Analg, 2001;92:12-18. 


\section{RESUMEN}

Gautam PL, Katyal S, Wander GS, Kaur H - Isquemia Miocárdica Silenciosa en Pacientes Sometidos a Prostatectomia Transuretral - Comparación entre Anestesia Subaracnóidea y Peridural

JUSTIFICATIVA Y OBJETIVOS: La isquemia miocárdica silenciosa fue recientemente relacionada al aumento de morbi-mortalidad cardíaca peri-operatoria. Hasta $41 \%$ de los pacientes con enfermedad coronariana conocida o con factores de riesgo cardíaco, sometidos a cirurgías no cardíacas, presentan isquemia peri-operatoria. Varios autores compararon las técnicas de anestesia regional y general, más ninguno comparó el impacto de diferentes técnicas de anestesia del neuro-eixo en la incidencia y duración de la isquemia miocárdica silenciosa. El objetivo de este estudio fue la de comparar dos técnicas diferentes de anestesia en el neuro-eixo (subaracnóideo versus. peridural) en pacientes edosos aleatoriamente seleccionados y sometidos a prostatectomia transuretral. Optaou-se por pacientes idosos por frecuentemente presenta enfermedad coronariana silenciosa o aparente. Outro factor importante que influenció la escoja, fue la sobrecarga de volumen y tremores causados por prostatectomia transuretral en esos pacientes promovendo desequilibrio entre el consumo y oferta de oxígeno.

MÉTODO: Participaron de este estudio 40 pacientes sometidos a prostatectomia, fueron estudiados en relación a la isquemia miocárdica silenciosa con la ayuda de un equipamiento Holter. La monitorización se inició 1 hora antes de la cirugía y prosiguió durante la misma y después por las próximas 24 horas. Los datos del Holter fueron analizados por un DSM modelo 300.

RESULTADOS: La incidencia general de isquemia miocárdica silenciosa en este estudio fue de $30 \%$. No fue establecida ninguna relación entre isquemia miocárdica silenciosa y tipo de anestesia. La mayor parte de los episodios de isquemia miocárdica ocurrió en el período pre-operatorio y no tuvieron relación con alteraciones hemodinámicas. No obstante, la incidencia y la gravidad de isquemia miocárdica silenciosa fue más alta en pacientes con altos contajes de Detsky, hipertensión arterial y anemia. Ningún paciente presentó efectos cardíacos adversos.

CONCLUSIONES: El tipo de anestesia no influenció la incidencia de isquemia miocárdica silenciosa. 